Pil E.A.

Theoretical possible scenarios for the development economic crises and the way out of them

State University of Aerospace Instrumentation (Russia, Saint-Petersburg)

doi: 10.18411/scienceconf-06-2021-14

\title{
Abstract
}

The article deals with the issues of classification different kinds of scenarios for countries using surfaces which their economy and population can occupy. Some formulas which are presented in this article allow us to calculate these surfaces. Present 2D-figures show us how economic crises can develop during time.

Keywords: economy, GDP, population, decrease, increase, economic crises, way out of crises.

Both growth and decline conditions in economy develop under the influence of a wide range of factors, among which is decrease or increase in the numbers of population due to certain objective reasons. To reveal the interrelation of predicted growth or decline in economy $e_{t}$, and hence predicted increase or decrease in the numbers of population $p_{t}$ table 1 was constructed to present all possible scenarios of dependence of variables $e_{t}$ and $p_{t}$ discussed. In the further discussion, economy is taken as the DGP of a country, which explains interchangeability of these terms in the context. Figures in Table 1 show one x-axis $Q_{t}$ instead of two $Q_{e t}$ and $Q_{p t}$ with varying scales and values, where $Q_{e t}$ and $Q_{p t}$ are given as percentages

Hereinafter in all the graphs of Table 1 , a dashed line indicates the $e_{t}$ parameter, and the full line indicates the $p_{t}$ parameter.

\section{Scenario 1}

In this scenario economy $e_{t}$ and population $p_{t}$ are constants not changing in time, i.e. $e_{t}=$ const and $p_{t}=$ const (see Row 1). Herein the first digit indicates the number of Row, the second, the number of column. Changes in $p_{t}$ and $e_{t}$ are described by the following linear equation: $y_{e t}=Q_{e t}$ and $y_{p t}=Q_{p t}$.

Cell 11 shows a variation of the number of population $Q_{p t}$ exceeding the economic variables $Q_{e t}$, i.e. $Q_{p t}>Q_{e t}$. Characterization of such condition requires introduction of a variable $Q_{p e t}$, which indicates the predicted difference of $Q_{p t}$ and $Q_{e t}$ and is calculated using the following formula (1).

$$
Q_{p e t}=Q_{p t}-Q_{e} \text {. }
$$

In this case, the higher value $Q_{p e t}$ is, the lower the standard of living of the population as consumption rates per capita decrease. To simplify, we consider consumption rates of goods, food and services to be equal with the upper and middle classes and low-income groups. Otherwise, higher values of $Q_{p e t}$ will mean upper classes wishing to at least preserve the current levels of consumption which, in condition of limited resources, means less goods and services for middle- and lower-income classes.

The following scenarios and variations appear possible in this case:

1. the population will be suffering lower standards of living with no opposition;

2. the population suffering lower standards of living will turn to sects and cults with resulting further worsening of their economic condition;

3. given any chance population will try to emigrate, legally or illegally, into more economically developed countries, primarily to those with low birth 
rates and high demand for workforce, mostly high-skilled and qualified, which is the current condition in European countries;

4. national government will be increasing the number of festivities and public entertainment to distract the population from worsening economic conditions;

5. national government will resort to the policy of confronting either the external or internal enemies to divert attention of the population from worsening economic conditions to 'public enemies' pretending them to be blamed with the existing decline. This scenario is proven by history of many modern countries;

6. national government will resort to the policy of stimulating consumption of alcoholic beverages by the population for such option is much more simple, quick, effective and efficient than construction of sports facilities and promotion of the healthy way of living. The population in this case is much easier to manipulate and they reveal less willingness and readiness to become actively involved in the country's political life. In addition, increased sales of spirits increase public revenues. The population will suffer degradation resulting in irreversible deterioration of the nation's gene pool;

7. national government will resort to the policy of befuddling the population of younger ages promoting sub-cultures to distract them from involvement in political life of the country;

8. national government will suppress opposition and pressure groups leaders using various options: court action, exile, imprisonment, asylums and similar institutions, physical elimination, forced emigration etc. In this latter case the leaders of opposition will be establishing centers abroad and using mass-media, global network and other legal and illegal means will be trying to undermine the existing regime under which their compatriots continue living;

9. national government will resort, either openly or using concocted reasons, to massive resettlements of politically active population from urban to rural localities to be reformed with manual labor;

10. national government will be increasing the share of military sector making it controlling and underlying the whole economy which will result in lower standards of living and high probability of military conflicts similar to those in Germany in the past century;

11. national government, to distract the country's population from democratic reforms, will initiate a military conflict with a weaker neighboring nation similar to that initiated by Iraq in 1990-1991;

12. national government seeking to improve the nation's economic condition will enter into a military conflict initiated by a third nation similar to that entered into by the US when high growth rates of the country's GDP were in direct relation to supplies of weapons and food to the parties in conflict;

13. population through the procedure of democratic election will impeach and depose the current government either accompanied by a deposition of the upper class as in Chile and Venezuela, or through a 'color revolution' as in the Ukraine and Georgia;

14. population will unroll a revolution to depose the existing regime and change the socio-economic formation as in Romania, Tunisia and other countries. 
Variations in interdependence of changes in theoretically predicted economy $e_{t}$ and

Table 1 population $p_{t}$

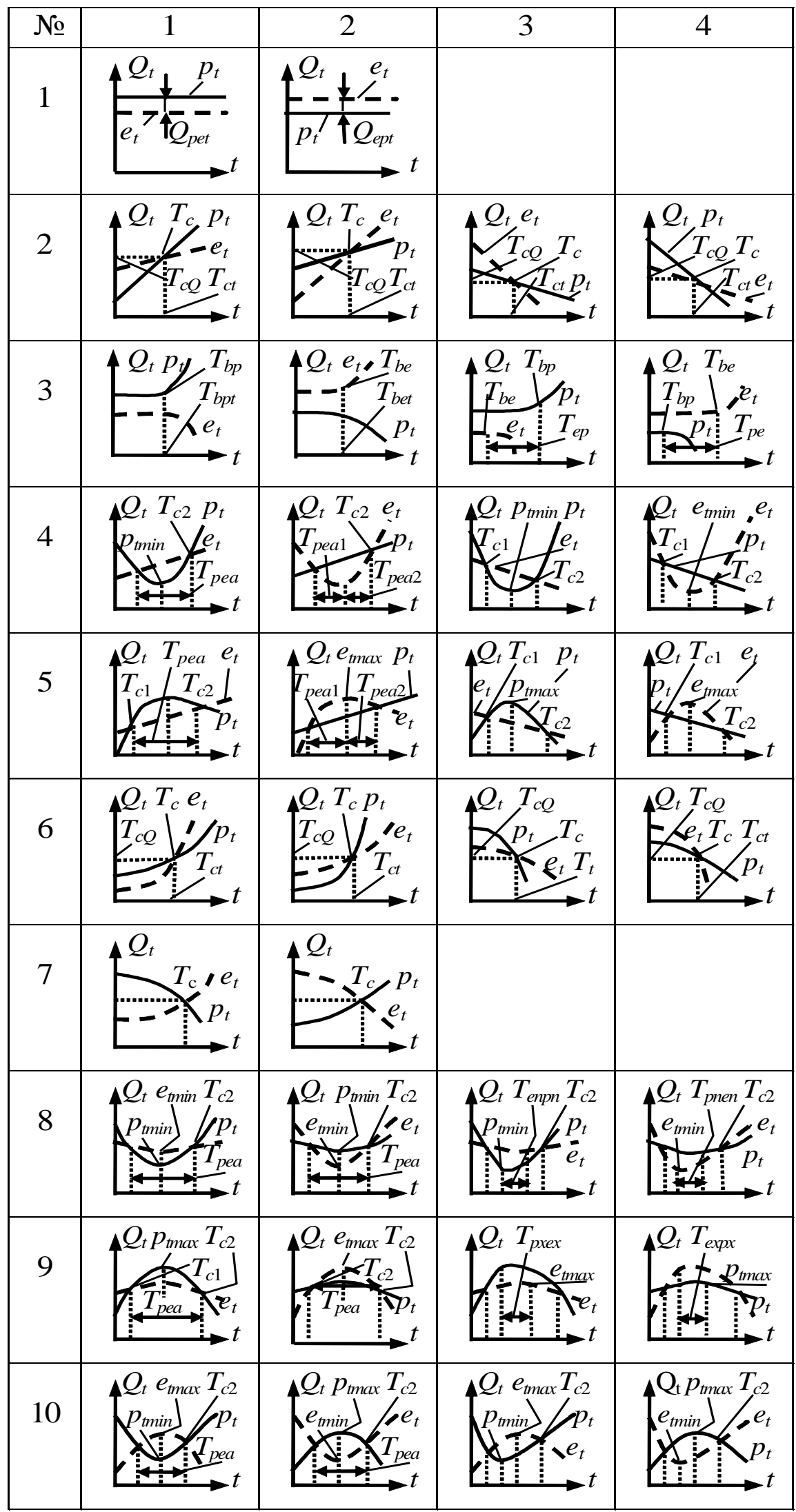

Cell 12 shows dependencies of economic indicators exceeding the number of population, i.e. $Q_{e t}>Q_{p t}$. In this case the standards of living of the population will be the higher, 
the higher is $Q_{e p t}$ value calculated by Formula (2) promoting well-being and prosperity of the population.

$$
Q_{e p t}=Q_{e t}-Q_{p t}
$$

Figures for variables $e_{t}$ and $p_{t}$ changing linearly can be characterized by the angle $\alpha$ of a given straight line to $\mathrm{x}$-axis. In the variation discussed $\alpha=0$ for all cases. In theory, the angle $\alpha_{t}$ can be within the range of $-90^{\circ} \leq \alpha_{t} \leq+90^{\circ}$. Thus, the sign of angle $\alpha_{t}$ either indicates growth (if positive) or decline (if negative) of the variables discussed.

\section{Scenario 2}

Row 2 presents variations in linear development of economy $e_{t}$ and the number of population $p_{t}$ with the figures of dependencies plotted intersecting in point $T_{c}$, with vales of $T_{c t}$ and $T_{c Q}$ along the coordinate axes $t$ and $Q_{t}$.

Within Scenario 2 the following variations in development are possible:

- $\quad$ the number of population $p_{t}$ is increasing at a faster rate than economy $e_{t}$, Cell 21 . In such case it is rational that the value $T_{c t}$ be maximum which will allow to delay the populating impoverishment;

- $\quad$ economy et is increasing at a faster rate than the number of population pt, Cell 22. In such case it is rational that the value Tct be the minimum. Such condition will allow reduction in time rates to improve the well-being of the population;

- economy et is decreasing at a faster rate than the number of population pt, Cell 23. In such case it is rational that the value Tct be the maximum. Such condition will allow avoiding fast rates of impoverishment while providing time for emergency measures to both increase the number of population and improve the economic conditions;

- the number of population pt is decreasing at a faster rate than economy et, Cell 24 . In such case it is rational that the value $T_{c t}$ be approximating the minimum. Such condition will allow minimizing time required to improve the well-being of the population. This variation has certain ethical implications since sensible and prudent population will refrain from improving their standards of living at the expense of the other population, the condition aggravated by decreased numbers of population causing reductions in labor force and hence resources for public goods. Thus, for this Scenario it appears rational to apply mechanized and automated processes of production, integration of innovative technologies etc. This scenario is applicable for countries with high rates of population, poor economic conditions and hence over-abundant workforce.

Since the figures plotted can be subdivided into classes and subclasses, with groups also identifiable within them, general conclusions are to be applicable to all these. This condition can be relied upon to avoid the necessity of discussing each line individually.

\section{Scenario 3}

Here scenario 3, which was inserted into this group, though it could have been included into the group of nonintersecting curves. In this scenario variables $e_{t}$ and $p_{t}$ are parallel to axis $t$ at first, and then they start changing according to a complex law in the points of bend of $T_{b e}$ and $T_{b p}$, where the tangent changes its direction, with similar coordinates (Cells 31 and 32), or with different coordinates (Cells 33 and 34). These Cells also show projections of points $T_{b e}$ and $T_{b p}$ to axis $t$, which are $T_{b e t}$ and $T_{b p t}$ respectively. Due to the fact that the coordinates of points $T_{b e}$ and $T_{b p}$ are different, we introduced here two new properties of $T_{e p}$ and $T_{p e}$, which are calculated with the formulas: $\left|T_{e p}\right|=T_{b e t}-T_{b p t} ;\left|T_{p e}\right|=T_{b p t}-T_{b e t}$. Here the values of $T_{e p}$ and $T_{p e}$ are considered according to the absolute value, as they can be the following: $T_{b p t}>T_{b e t} ; T_{b p t}<T_{b e t}$ or $T_{b p t}=T_{b e t}$.

If the economy $e_{t}$ and population $p_{t}$ change their linear law to a more complex one at the same time, i.e. $T_{b e t}=T_{b p t}$, as it is presented in Cells 31 and 32, let us add new parameters in order to make conclusions: $\pm \Delta e_{t} ; \pm \Delta p_{t} ; \Delta t_{e}$ and $\Delta t_{p}$, where $\pm \Delta e_{t}$ is growth or recession of the economy in the time period $\Delta t_{e}$ in question, unit, and $\pm \Delta p_{t}$ is growth or decrease of population in the time period $\Delta t_{p}$ in question, pers. This means that we need to know how variables $e_{t}$ and $p_{t}$ will 
increase or decrease within the time period $\Delta t_{e}$ or $\Delta t_{p}$. In this case values $\Delta t_{e}$ and $\Delta t_{p}$ should be taken as equal, i.e. $\Delta t_{e}=\Delta t_{p}$. In effect it is desirable to assume that values $\Delta t_{e}$ and $\Delta t_{p}$ are equal to one, i.e. one hour, day, week, month or year, depending on specific conditions and the prospect of timely receiving accurate information on changes taking place in the economy or population.

Thus, we can note down the following three formulas for Cell 31:

1. $\Delta p_{t}-\left|-\Delta e_{t}\right|=0$

2. $\Delta \mathrm{pt}-|-\Delta \mathrm{et}|>0$;

3. $\Delta \mathrm{pt}-|-\Delta \mathrm{et}|<0$.

As for Cell 32, we can note down the following:
4. $\Delta \mathrm{et}-|-\Delta \mathrm{pt}|=0$;
5. $\Delta \mathrm{et}-|-\Delta \mathrm{pt}|>0$;
6. $\quad \Delta \mathrm{et}-|-\Delta \mathrm{pt}|<0$.

For the sake of simplicity here the + sign was omitted.

In Cells 31 and 32 in question there are the following two evolution scenario possible:

1. When variables $e_{t}$ and $p_{t}$ change by the same value, i.e. when $\left| \pm \Delta e_{t}\right|=\left| \pm \Delta p_{t}\right|$. If the values are equal $\left| \pm \Delta e_{t}\right|=\left| \pm \Delta p_{t}\right|$, it is desirable for Cell 31 that the value of point $T_{b p t}$ be at its maximum, as in this case the population's wealth will decrease far in the future. As for values $\left| \pm \Delta e_{t}\right|$ and $\left| \pm \Delta p_{t}\right|$, here they must be at their minimum, as in this case the population's wealth will decrease very slowly;

2. When variables $e_{t}$ and $p_{t}$ change according to different laws, i.e. $\left| \pm \Delta e_{t}\right| \neq\left| \pm \Delta p_{t}\right|$. Here it is desirable for variables $e_{t}$ and $p_{t}$ in box 31 that the sum of values $\left|-\Delta e_{t}\right|$ and $\Delta p_{t}$ be at its minimum $\left(\left|-\Delta e_{p}\right|+\Delta p_{t}\right) \rightarrow \min$. In this case population growth and simultaneous recession of the country's economy will take place more smoothly. As for Cell 32, here it is desirable to have maximum $\Delta e_{t}$ and $\left|-\Delta p_{t}\right|$ values, as in this case population's wealth will increase in a minimum time period.

For Cells 33 and 34 there are the following two evolution scenarios possible:

1. If the economy starts falling earlier than population starts growing, it is desirable that the value of $T_{e p}$ tend to the maximum, i.e. $T_{e p} \rightarrow \max$, as in this case population will slide into poverty slower (Cell 33);

2. If the economy starts improving later than population starts decreasing, it is desirable that the value of $T_{p e}$ tend to the minimum, i.e. $T_{e p} \rightarrow \min$, as in this case growth of population's income may have a good impact on birth rate (Cell 34).

\section{Scenario 4}

Row 4 shows variables $e_{t}$ or $p_{t}$, with the difference as compared to the above that either variable is a curve which at first reduces to its minimum $T_{\text {emin }}\left(T_{p m i n}\right)$, then increasing and intersecting with the straight line twice. In Cells 41 and 42 the straight lines discussed increase, in Cells 43 and 44 they decline.

The parameters are to have the following values.

For parameters $\Delta e_{t}$ and $\Delta p_{t}$ :

- Cells 41, 43:

before the minimum point: $-\Delta p_{t} \rightarrow \max$;

after the minimum point: $\Delta p_{t} \rightarrow \min$.

- Cells 42, 44:

before the minimum point: $-\Delta e_{t} \rightarrow \min$;

after the minimum point: $\Delta e_{t} \rightarrow \max$.

For parameters $T_{p e a}$ : 
- $\quad$ Cell 41: $T_{\text {pea }} \rightarrow \max , T_{\text {pea1 }} \rightarrow \max , T_{\text {pea } 2} \rightarrow \max$;

- Cell 42: $T_{\text {pea }} \rightarrow \min , T_{\text {peal }} \rightarrow \min , T_{\text {pea } 2} \rightarrow \min$;

- Cell 43: $T_{\text {pea }} \rightarrow \max , T_{\text {peal }} \rightarrow \max , T_{\text {pea } 2} \rightarrow \max$;

- Cell 44: $T_{\text {pea }} \rightarrow \min , T_{\text {pea1 }} \rightarrow$ min,$T_{\text {pea } 2} \rightarrow$ min .

In this Scenario in choosing either maximum or minimum values of the parameters discussed a certain ambiguity arises in interpretation. For instance, if in Cell 44 parameter $T_{c t 1}$ is a maximum, the economic well-being of the population will be decreasing during a long period to shift to fast increase. If the value of parameter $T_{c t 1}$ is a minimum, the economic wellbeing of the population be decreasing fast and then increasing fast. The latter option, with $T_{\text {ept } 1} \rightarrow \min$ is deemed more rational.

To calculate the new parameters Formula (3) can be used

$$
T_{p e a}=T_{p e a 1}+T_{p e a 2}=T_{c t 2}-T_{c t 1}=\left(T_{p t m i n}-T_{c t 1}\right)+\left(T_{c t 2}-T_{p t m i n}\right),
$$

where $T_{c a 1}, T_{p t m i n}$ etc. are coordinates of respective points on axis $t$.

\section{Scenario 5}

This Scenario is similar to Scenario 5 with a curve maximum. The curve first rises to its maximum $T_{\text {emax }}\left(T_{\text {pmax }}\right.$ ), and then slopes down and intersects with the straight line twice. In Cells 51 and 52 the straight lines discussed first slope upwards and in Cells 53 and 54 downwards.

The parameters are to have the following values:

For parameters $\Delta e_{t}$ and $\Delta p_{t}$ :

- Cells 51, 53:

before the maximum point: $\Delta p_{t} \rightarrow \min$;

after the maximum point: $-\Delta p_{t} \rightarrow \max$.

- Cells 52, 54:

before the maximum point: $\Delta e_{t} \rightarrow \max$;

after the maximum point: $-\Delta e_{t} \rightarrow$ min .

For parameters $T_{\text {pea }}$ :

- Cell 51: $T_{\text {pea }} \rightarrow \min , T_{\text {peal }} \rightarrow \min , T_{\text {pea } 2} \rightarrow \min$;

- Cell 52: $T_{\text {pea }} \rightarrow \max , T_{\text {peal }} \rightarrow \max , T_{\text {pea } 2} \rightarrow \max$;

- Cell 53: $T_{\text {pea }} \rightarrow \min , T_{\text {peal }} \rightarrow \min , T_{\text {pea } 2} \rightarrow \min$;

- $\quad$ Cell 54: $T_{\text {pea }} \rightarrow \max , T_{\text {peal }} \rightarrow \max , T_{\text {pea } 2} \rightarrow \max$.

The parameters introduced can be calculated with the Formula (4)

$$
T_{p e a}=T_{p e a 1}+T_{p e a 2}=T_{c t 2}-T_{c t 1}=\left(T_{p t \max }-T_{c t 1}\right)+\left(T_{c t 2}-T_{p t \max }\right),
$$

where $T_{\text {peal }}, T_{\text {ptmax }}$ etc. are coordinates of respective points on axis $t$.

\section{Scenarios 6 and 7}

These Scenarios are characterized by figures plotted for $e_{t}$ and $p_{t}$ intersecting in one point $T_{c}$.

The parameters are to have the following values:

- Cell 61: $T_{c t} \rightarrow \min , \Delta e_{t} \rightarrow \max , \Delta p_{t} \rightarrow \min$;

- $\quad$ Cell 62: $T_{c t} \rightarrow \max , \Delta e_{t} \rightarrow \max , \Delta p_{t} \rightarrow \min$;

- Cell 63: $T_{c t} \rightarrow \min ,-\Delta e_{t} \rightarrow \min ,-\Delta p_{t} \rightarrow \max$;

- $\quad$ Cell 64: $T_{c t} \rightarrow \max ,-\Delta e_{t} \rightarrow \min ,-\Delta p_{t} \rightarrow \max$;

- Cell 71: $T_{c t} \rightarrow \min , \Delta e_{t} \rightarrow \max ,-\Delta p_{t} \rightarrow \max$;

- Cell 72: $T_{c t} \rightarrow \max ,-\Delta e_{t} \rightarrow \min , \Delta p_{t} \rightarrow \min$. 
The below three Scenarios 8, 9 and 10 show curves intersecting in two points. Here variables $e_{t}$ and $p_{t}$ can be characterized by either coincident minimums and maximums, i.e. $T_{p t \min }=T_{\text {etmin }}$ etc. (Cells 81, 82, 91, 92, 101 and 102), or different minimums and maximums, i.e. $T_{p t \text { min }}>T_{\text {etmin }}$ or $T_{p t \text { min }}<T_{\text {etmin }}$ etc. (Cells 83, 84, 93, 94, 103 and 104).

\section{Scenario 8}

Description of Scenarios given in Row 8 and in Rows 9 and 10 requires plotting figures for variables $e_{t}$ and $p_{t}$ with maximums and minimums both on one straight line and displaced from it, hence, the below parameters are to be introduced:

1. $T_{p n e n}$ is the difference between the coordinates of points $T_{p t m i n}$ and $T_{e t m i n} . \mathrm{m.u}$;

2. $T_{\text {enpn }}$ is the difference between the coordinates of points $T_{p t m a x}$ and $T_{\text {etmax }}$. m.u;

3. $T_{\text {pxex }}$ is the difference between the coordinates of points $T_{\text {ptmax }}$ and $T_{\text {etmax }}$. m.u;

4. $T_{\text {exp }}$ is the difference between the coordinates of points $T_{\text {etmax }}$ and $T_{p t m a x}$. m.u;

5. $T_{p x e n}$ is the difference between the coordinates of points $T_{p t m a x}$ and $T_{\text {etmin. }} . \mathrm{m} . \mathrm{u}$;

6. $T_{\text {expn }}$ is the difference between the coordinates of points $T_{\text {etmax }}$ and $T_{\text {ptmin. }}$. m.u;

7. $T_{p \text { nex }}$ is the difference between the coordinates of points $T_{p t \text { tmin }}$ and $T_{\text {etmax }}$. m.u;

8. $T_{\text {enpx }}$ is the difference between the coordinates of points $T_{\text {etmin }}$ and $T_{p t m a x}$. m.u.

The above eight parameters demonstrate shifting vertexes in figures of $e_{t}$ and $p_{t}$, with lower values of index enpx indicating the first intersection point be a curve, in this case the coordinate of the minimum point of variable $e_{t}$, and the second intersection point being the maximum point of variable $p_{t}$, i.e. $T_{\text {etmin }}>T_{\text {ptmax }}$.

Row 8 shows variations of figures for variables $e_{t}$ and $p_{t}$ as two curves with minimums and intersections in two points. Here in Cells 81 and 82 their minimums lay along the same straight line, while in cells 83 and 84 they are shifted relatively to each other by value of $T_{\text {enpn }}$ (Cell 83) or by value of $T_{\text {pnen }}($ Cell 84).

The parameters are to have the following values.

For parameters $\Delta e_{t}$ and $\Delta p_{t}$ :

- Cells 81, 82, 83 and 84:

before the point $T_{e p}$ and $T_{b p}:-\Delta e_{t} \rightarrow \min ,-\Delta p_{t} \rightarrow \max$;

after the point $T_{e p}$ and $T_{b p}: \Delta e_{t} \rightarrow \max , \Delta p_{t} \rightarrow \min$.

For parameter $T_{\text {pea }}$ :

- Cells 81 and 83: $T_{\text {pea }} \rightarrow \max$;

- Cells 82 and 84: $T_{\text {pea }} \rightarrow \min$.

For parameters $T_{\text {enpn }}$ and $T_{\text {pnen }}$ :

- Cells 83 and 84: at $T_{b e t}>T_{b p t}, T_{\text {pnen }} \rightarrow$ min ;

at $T_{b e t}<T_{b p t}, T_{\text {enpn }} \rightarrow \max$.

For parameters $Q_{\text {enpn }}$ and $Q_{\text {pnen }}$ :

- Cells 81 and 83: $Q_{\text {enpn }} \rightarrow \max$;

- Cells 82 and 84: $Q_{\text {pnen }} \rightarrow \min$.

\section{Scenario 9}

Row 9 shows variations in figures for variables $e_{t}$ and $p_{t}$ as two curves with maximums and intersections in two points. Here in Cells 91 and 92 their maximums lie along the same straight line, while in Cells 93 and 94 they are shifted relatively to each other by value of $T_{\text {pxex }}$ (Cell 93) or by value of $T_{\text {expx }}$ (Cell 94).

The parameters are to have the following values.

For parameters $\Delta e_{t}$ and $\Delta p_{t}$ :

- Cells 421, 422, 423 and 424:

before the points $T_{e p}$ and $T_{b p}: \Delta e_{t} \rightarrow \max , \Delta p_{t} \rightarrow \min$;

after the points $T_{e p}$ and $T_{b p}:-\Delta e_{t} \rightarrow \min -\Delta p_{t} \rightarrow \max$. 
For parameters $T_{\text {epx }}$ and $T_{p e x}$ :

- Cells 421 and 423: $T_{p e x} \rightarrow \min$;

- $\quad$ Cells 422 and 424: $T_{e p x} \rightarrow \max$.

For parameters $T_{\text {exp }}$ and $T_{p x e x}$ :

- $\quad$ Cell 423: at $T_{b e t}>T_{b p t}, T_{p x x} \rightarrow \max$;

at $T_{b e t}<T_{b p t}, T_{e x p x} \rightarrow \min$.

- $\quad$ Cell 424: at $T_{\text {bet }}>T_{b p t}, T_{p x e x} \rightarrow \min$;

at $T_{\text {bet }}<T_{b p t}, T_{\text {exp }} \rightarrow \max$.

For parameters $Q_{\text {enpn }}$ and $Q_{\text {pnen }}$ :

- Cells 421 and 423: $Q_{\text {enpn }} \rightarrow \max$;

- $\quad$ Cells 422 and 424: $Q_{\text {pnen }} \rightarrow$ min .

\section{Scenario 10}

Row 10 shows variations in figures for variables $e_{t}$ and $p_{t}$ as two curves with maximums and intersections in two points. Here in Cells 101 and 102 their maximums and minimums lie along the same straight line, while in Cells 103 and 104 they are shifted relatively to each other by value of $T_{p x e x}$ (Cell 103) or by value of $T_{\text {expx }}$ (Cell 104).

The parameters are to have the following values.

For parameters $\Delta e_{t}$ and $\Delta p_{t}$ :

- Cells 101 and 103:

before the points $T_{e p}$ and $T_{b p}: \Delta e_{t} \rightarrow \max ,-\Delta p_{t} \rightarrow \max$;

after the points $T_{e p}$ and $T_{b p}:-\Delta e_{t} \rightarrow \min , \Delta p_{t} \rightarrow \min$.

- Cells 101, 102 and 104:

before the points $T_{e p}$ and $T_{b p}:-\Delta e_{t} \rightarrow \min , \Delta p_{t} \rightarrow \min$;

after the points $T_{e p}$ and $T_{b p}: \Delta e_{t} \rightarrow \max ,-\Delta p_{t} \rightarrow \max$.

For parameters $T_{e p x}$ and $T_{p e x}$ :

- Cells 101 and 103: $T_{p e x} \rightarrow \max$;

- Cells 102 and 104: $T_{e p x} \rightarrow$ min .

For parameters $T_{\text {enpx }}$ and $T_{\text {pnex: }}$ :

- Cells 433: at $T_{b e t}>T_{b p t}, T_{\text {pnex }} \rightarrow \max$;

at $T_{b e t}<T_{b p t}, T_{\text {pnex }} \rightarrow \max$.

- Cells 434: at $T_{b e t}>T_{b p t}, T_{e n p x} \rightarrow \min$;

at $T_{b e t}<T_{b p t}, T_{e n p x} \rightarrow \min$.

For parameters $Q_{p x e n}$ and $Q_{\text {expn }}$ :

- Cells 101 and 103: $Q_{\text {expn }} \rightarrow \max$;

- Cells 102 and 104: $Q_{\text {pxen }} \rightarrow \min$.

In addition to these scenarios variables $e_{t}$ and $p_{t}$ they have 'intersection point' $T_{c}$ (rows 7 10). Since intersection point $T_{c}$ is characterized by same coordinates for figures of $e_{t}$ and $p_{t}$ it is termed here 'the point of equilibrium'. In this point the population of a given country consumes the whole of food, other goods and services produced within the country.

These variables $e_{t}$ and $p_{t}$ can be also merging into one line or curve together. So such merging figures allow us to introduce a new term 'equilibrium of economy and population', i.e. a condition when the values of the country's GDP and the number of population are equal $Q_{e t i}=Q_{p t i}$. 

population.

Now let us introduce the following three definitions to equilibrium of the economy and

Theoretically predicted equilibrium in economy and population refers to equality of $Q_{\text {eti }}=$ $Q_{p t i}$, when the population consumes the total amount of food, other goods and services in a period of time $Q_{e t i}$, which are produced during the same period of time domestically without any losses and imports $Q_{\text {eim. }}$.

$Q_{e t i}$ and $Q_{p t i}$ are taken here for $100 \%$. Here, the universal expression will be formulated as $Q_{e t i}=Q_{p t i}+Q_{e i m}$ at $Q_{e i m}=0$. Hence, the total of $100 \%$ of food, other goods and services will be consumed by $100 \%$ of population in a period of time. It follow from here, that every person consumes equal numbers of food, other goods and services despite varying incomes.

Losses here refer primarily to losses resulting from 'wear and tear' in collection, transportation, storing and processing of agricultural produce and their delivery to the endconsumer and later in the process of home food procession, cooking and serving. Losses in other consumer goods refer to faulty and substandard items, damage in loading-unloading operations, transportation etc. after which they are to be disassembled and/or utilized. Another assumption in relation to non-foods is that they will be used during the period stated with no repair required after which time they are to be replaced. Services are assumed to fully correspond to the service wanted and ordered by the customer and provided in stated time with the quality required.

Practically attainable equilibrium of economy and population refers to equality $Q_{\text {epi }}=$ $Q_{p p i}$, when the population consumes in a period of time the total amount of food, other goods and services which are produced during the same period of time domestically given losses $Q_{\text {etl }}$ and without any imports.

In this case the following expressions are true: $Q_{e p i}>Q_{\text {eti }}$ or $Q_{e p i}=Q_{e t i}+Q_{e t l}$.

Effective equilibrium of economy and population refers to equality $Q_{\text {eri }}=Q_{\text {pri }}$, when the population consumes in a period of time the total amount of food, other goods and services which are produced during the same period of time domestically given losses $Q_{\text {etl }}$ and the difference between the effective and theoretically predicted $Q_{\text {eti }}$ is compensated by imports $Q_{\text {eim }}$.

In this case the following expressions are true: $Q_{e t l}=Q_{e i m}, Q_{e r i}=Q_{p r i}+Q_{e i m}$.

It should be noted that the amount of foods, other goods and services to be consumed is to be scientifically justified by estimated and practically proven standards and norms $Q_{s n}$ for the period discussed or a point in time in a given country. Otherwise, the population will either start gaining or losing weight, in terms of the amount of food consumption. In terms of non-food products (durables) disequilibrium in consumption will result in either increased numbers of waste and entities to recycle or utilize them, or increased amounts of exports to less developed countries; insufficient amounts of outputs will cause shortages.

Thus, the expression here can be given as $Q_{s n}=Q_{e s n} / Q_{p i}$, where $Q_{s n}$ is scientifically justified amount of consumed food, non-food goods and services, in measurement units; $Q_{e s n}$ is the amount of food, non-food goods and services produced for scientifically justified consumption, in measurement units; $Q_{p i}$ is the number of population in a country, number of people.

Another essential condition in this case is the necessary requirement for the national government to possess strategic food and commodities reserve stock, various metals and other raw materials etc., and mothballed facilities and equipment for production for military purposes for survival in extreme cases of poor crops, natural disasters and military actions. Mothballed facilities for military purposes are affordable for either economically advanced nations, or totalitarian regimes.

If the amount of food, non-food goods and services produces in a period of time $Q_{p t i}$ exceeds the scientifically justified calculations $Q_{s n}\left(Q_{p t i}>Q_{s n}\right)$, the surpluses can be used in the following ways:

- exported; 
- $\quad$ added to the nation's strategic reserve stock;

- $\quad$ exported and added to the nation's strategic reserve stock.

A scenario is possible in this case when given $Q_{p t i}=Q_{s n}$ in countries with low levels of wages and salaries and standards of living or those suffering a sharp decline in economy population will be unable to consume all of the food, non-food and services output produced, these surpluses will be exported to countries with higher standards of living increasing the country's exports revenues, if they will be bought there [1].

These Figures in Table 1 became the basis for calculations which allowed us to find different kinds of way out of economic crises [2].

\section{***}

1. Pil E.A. The Theory of Economic Crises. - St.-Petersburg: Asterion, 2017. - 1064 p.

2. Pil E.A. The ways out of the economic crises using analyse 3D figures Veu and variable X5eu // Norway Journal of development of the International Science. №54/2021. Vol.2 64 p - P. 16-23 